\title{
Correction to: Systematic expression analysis of Hox genes at adulthood reveals novel patterns in the central nervous system
}

\author{
Bertrand Hutlet ${ }^{1} \cdot$ Nicolas Theys $^{1} \cdot$ Cécile Coste $^{1,2} \cdot$ Marie-Thérèse Ahn ${ }^{1} \cdot$ Konstantin Doshishti-Agolli ${ }^{1}$. \\ Benoît Lizen ${ }^{1} \cdot$ Françoise Gofflot ${ }^{1}$
}

Published online: 18 March 2021

(c) Springer-Verlag GmbH Germany, part of Springer Nature 2021

\section{Correction to: Brain Struct Funct (2016) 221:1223-1243 https://doi.org/10.1007/s00429-014-0965-8}

"It has been noted that in the original version of Figure 2, the image shown in panel 2D incorrectly displayed a zoomed version of panel $2 \mathrm{C}$. This has been corrected in the revised figure in order to show the intended image for panel 2D."

The original article can be found online at https://doi.org/10.1007/ s00429-014-0965-8.

Françoise Gofflot

francoise.gofflot@uclouvain.be

1 Institut des Sciences de la Vie, Université catholique de Louvain, 1348 Louvain-La-Neuve, Belgium

2 Present Address: Laboratory of Developmental Neurobiology, GIGA-Neurosciences Research Center, University of Liège, 4000 Liège, Belgium 


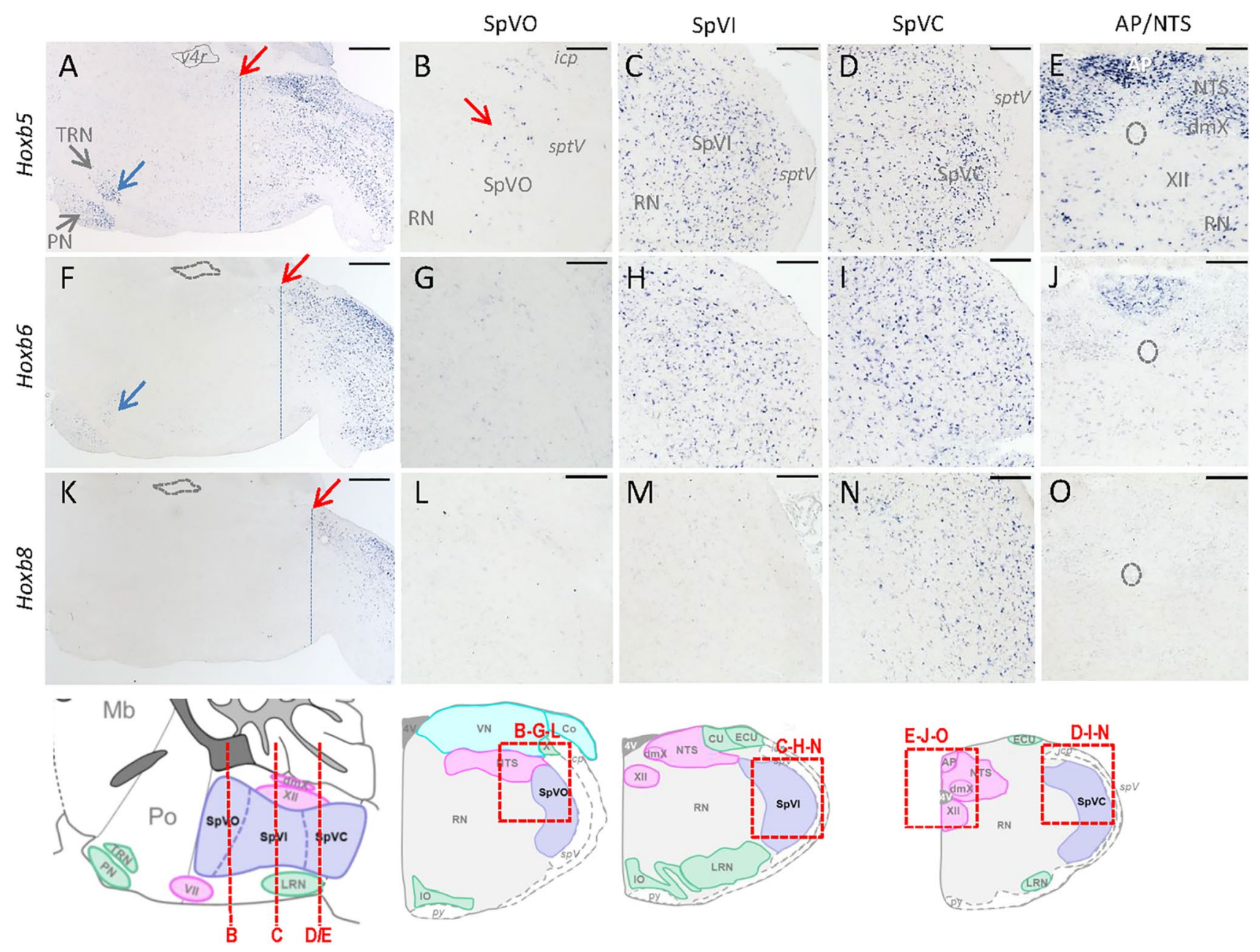

Fig. 2 Hox gene expression in the hindbrain of adult C57B1/6 J mice. In situ hybridization of Hoxb5, Hoxb6 and Hoxb8 on sagittal and coronal cryosections of adult mouse hindbrain. Schematic views of sagittal and coronal hindbrain sections in the bottom row indicate the localization of pictures. a, f, $\mathbf{k}$ On sagittal sections, a border of expression is observed at distinct antero-posterior levels for the three genes (dotted blue line). The anterior limit is clearly visible on the dorsal region (red arrows). Anterior to this border, expression is also detected in discrete areas, such as the pontine nuclei (PN) and the tegmental reticular nuclei (TRN) (blue arrows). b-d, $\mathbf{g}-\mathbf{i}, \mathbf{l}-\mathbf{n}$ On coronal sections, expression within the antero-posterior subnuclei constituting the spinal trigeminal nucleus $(\mathrm{SpV})$ showed nested overlapping domains with sequential anterior boundaries. Hoxb5 is expressed in the oral part (SpVO red arrow), the intermediate part (SpVI) and

the caudal part (SpVC). Hoxb6 is expressed in the SpVI and SpVC, and Hoxb8 is expressed only in SpVC. e, j, o On coronal sections, expression within the dorsal region of the caudal adult hindbrain showed partially overlapping patterns. Hoxb5 is expressed in the area postrema (AP), the nucleus of the solitary tract (NTS) and the dorsal motor nucleus of the vagus nerve $(\mathrm{dmX})$. Hoxb6 is expressed only in the AP and scattered cells in the NTS, while no labelling is detected for Hoxb8. Dotted circles mark the ventricle. On sagittal sections, anterior is on the left; on coronal sections, medial is on the left. Fibre tracts are in italic. $v 4 r$ fourth ventricle, lateral recess; $s p t V$ spinal tract of the trigeminal nerve; icp inferior cerebellar peduncle; $R N$ reticular nucleus; XII hypoglossal nucleus. Scale bar $500 \mu \mathrm{m}$ in $\mathbf{a}, \mathbf{f}$ and $\mathbf{k}$; $200 \mu \mathrm{m}$ in others

Publisher's Note Springer Nature remains neutral with regard to jurisdictional claims in published maps and institutional affiliations. 